\title{
Dynamics of 2014 Ebola Epidemic in West Africa
}

\author{
Mutuguta John*, Rotich Titus ${ }^{\dagger}$ Chepkwony Isaac ${ }^{\ddagger}$
}

\begin{abstract}
Ebola virus disease is transmitted from human to human through physical contact of body fluid of infected or dead case. It can also be transmitted indirectly through contact with contaminated surfaces. This risk is however low and can be minimised through cleaning the surfaces by the use of disinfectants. In this paper we investigate the transmission dynamics of the 2014 Ebola virus disease in west Africa. We use a four compartmental network of Susceptible individuals (S), Exposed individuals (E), Infectious individuals (I) and the Removed individuals $(\Re)$ without demographic factors. The removed compartment is further split into two compartments i.e the recovered individuals (R) and the dead and buried individuals (D). We give the mathematical model that describe the transmission dynamics of the 2014 Ebola virus disease in West Africa. The results of the model analysis indicate that the threshold value for the transmission dynamics equals the relative removal rate. It is shown that provided that the susceptible ratio is kept below the relative removal rate then the epidemic will not occur.
\end{abstract}

Keywords; Ebola virus disease, basic reproduction number, epidemic threshold

Mathematics subject classification: 97M60, 00A71, 46N70

\section{Introduction}

Infection with Ebola virus causes a severe disease in humans known as Ebola virus disease. There are five species of the genus Ebolavirus, these are Zaire Ebolavirus, Sudan Ebolavirus, Reston Ebolavirus, Tai forest Ebolavirus and Bundibugyo Ebolavirus. The 2014 outbreak of Ebola virus disease in west Africa was caused by the Zaire Ebolavirus. Ebola virus is a Biosafety Level Four (BSL-4) pathogen and requires special containment measures and barrier protection particularly for healthcare workers. The virus can survive in liquid or dried material for many days [4]. They are inactivated by gamma irradiation, heating for 60 minutes at $60^{\circ} \mathrm{C}$ or boiling for five minutes and are sensitive to sodium hypochlorite bleach and other disinfectants. Freezing or refrigeration will not inactivate the Ebola virus. The incubation

\footnotetext{
*Murang'a University of Technology, Department of Mathematics and Actuarial Science, email:jmutuguta@mut.ac.ke

${ }^{\dagger}$ Moi University, Department of Mathematics and Physics, email: tisesko@yahoo.com

${ }^{\ddagger}$ Kenyatta University, Department of Mathematics, email: ichepkwony@gmail.com
} 
period for the Ebola virus disease is 2 to 21 days. The case-fatality ratio of the Zaire Ebolavirus infections is estimated to be between $44 \%$ and $90 \%$ [4]. Ebola virus is highly transmissible by direct contact with infected blood, secretions, tissue organs and other body fluids from dead or living infected persons. The principle mode of transmission in human outbreaks is person-to-person transmission through direct contact with a symptomatic or dead Ebola virus disease case. Burial ceremonies and handling of dead bodies play an important role in transmission [6].

The 2014 Ebola virus disease outbreak is the largest outbreak of the Ebola virus disease ever which had an exponential growth with a doubling rate of 34.8 days [5]. The outbreak begun in December 2013 in Guinea and spread to Liberia in March 2014, Sierra Leone in May 2014 and later to Nigeria and Senegal and as of $2^{\text {nd }}$ October 2014, 8,997 cases of Ebola virus disease including 4,493 deaths had been reported by the World Health Organization (WHO) in seven countries i.e. Guinea, Liberia, Sierra Leone, Nigeria, Senegal, Spain and USA [4]. As the epidemic evolved and more staff deployed in the affected countries to support the epidemic control, the risk of importation of Ebola virus disease cases to European Union and other countries increased. However, the risk of Ebola virus from an Ebola virus disease patient arriving in the EU as a result of a planned medical evacuation was considered to be low when appropriate measures were strictly adhered to but could not be excluded in exceptional circumstances [4]. The key parameter that describe the spread of the infection is the basic reproduction number $\left(R_{0}\right)$. If $R_{0}<1$, then the epidemic will stop and the Ebola virus will go extinct. However, if $R_{0}>1$ then the epidemic will grow. The estimates for $R_{0}$ from the outbreaks of Congo in 1995 and Uganda in the year 2000 ranged between 1.3 and 2.7 [1].

Our goal in this paper is to understand the dynamics of the 2014 Ebola virus epidemic in west Africa. Since Ebola virus disease is a communicable disease that spreads much faster than the natural demographic processes, we will model the 2014 epidemic using a four compartmental network of Susceptible individuals (S), Exposed individuals (E), Infectious individuals (I) and the Removed individuals $(\Re)$ without demographic factors. The removed compartment will further be split into two compartments i.e the recovered individuals $(R)$ and the dead and buried individuals (D). We will then estimate the reproduction number in the absence of control measures.

The framework of the model is given in figure 1

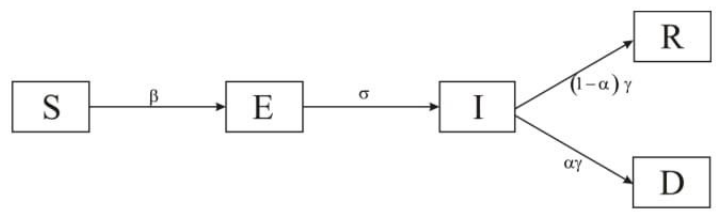

Figure 1: Flow chart describing the dynamics of the Ebola Virus Disease 


\section{The Mathematical Model}

The mathematical model for the system is given by;

$$
\begin{aligned}
& \frac{d S}{d t}=-\beta(t) S I \\
& \frac{d E}{d t}=\beta(t) S I-\sigma E \\
& \frac{d I}{d t}=\sigma E-\gamma I \\
& \frac{d R}{d t}=(1-\alpha) \gamma I \\
& \frac{d D}{d t}=\alpha \gamma I
\end{aligned}
$$

where $\alpha$ is the case fatality rate, $\sigma$ is the progression rate from exposed to infectious compartments, $\gamma$ is the removal rate and $\alpha$ is the disease mortality rate.

In the above SEIR model, susceptible individuals in contact with the virus enter the exposed compartment $E$ at a rate $\beta$. Individuals in this compartment are assumed to be asymptomatic and uninfectious but undergoes a mean incubation period of $\frac{1}{\sigma}$ before progressing to the infectious class $I$ at a rate $\sigma$. Infectious individuals progress to removed compartment at a rate $\gamma$. The Removed compartment consist of both the recovered individuals $\mathrm{R}$ and the individuals who are dead and buried D.

The transmission rate in the absence of control measures is given by $\beta(t)=\beta$. After the introduction of control measures at $\tau \leq t$, the transmission rate decays as a function of time at a rate $k$. Thus

$$
\beta(t)=\beta e^{-k(t-\tau)}
$$

\subsection{Basic Reproduction Number $R_{0}$}

The basic reproduction number is the most important quantity in infectious disease modeling. It is among the quantities most urgently estimated for emerging infectious diseases in outbreak situations and its value provides insights when designing control interventions for established infections. It has been shown that the basic reproduction number is mathematically characterised by regarding infections as a demographic process where producing offspring is not seen as giving birth in the normal demographic sense but as causing a new infection through transmission. This process is termed epidemiological birth. In natural way this leads to viewing the infection process in terms of consecutive generations of infected individuals [3]. In epidemic modeling, generations are the waves of secondary infections that flow from each previous infection. Thus the first generation of an epidemic comprise of all the secondary infections that result from the infectious contact with the index case who is regarded as generation zero. Therefore if $R_{i}$ denotes the reproduction number of the $i^{\text {th }}$ generation, then $R_{0}$ will denote the number of infections generated by the index case [2]. These numbers are small and are subject to sampling errors, we however determine the mean value hence we find an average over a large number of epidemics. The first step when determining this number is to construct 
the next generation matrix (NGM) of the epidemic. In the construction of the next generation matrix, one begins by identifying those equations in the system that describe the production of new infections and the changes in state among infected individuals. This set of equations is referred to as the infected subsystem. We then linearlise the system over the disease free equilibrium (DFE), the linearlization indicates that $R_{0}$ characterises the potential for the initial spread of the infectious agent when it is introduced into a fully susceptible population and therefore we assume that the change in the susceptible population is negligible during the initial spread.

The right hand side of the system is split into the transmission matrix $(\mathrm{T})$ and the transition matrix $(\mathrm{V})$. The basic reproduction number is then obtained as the spectral radius of the next generation matrix $\left(T V^{-1}\right)$ i.e $R_{0}=\rho\left(T V^{-1}\right)$ [3].

Consider the system 1 above. Since the system is closed and $R+D=1-(S+E+I)$ and that $R$ and $D$ are partitioned by the constant $\alpha$ then the system is a three dimensional system and may be expressed as;

$$
\begin{aligned}
\frac{d E}{d t} & =\beta(t) S I-\sigma E \\
\frac{d I}{d t} & =\sigma E-\gamma I \\
\frac{d S}{d t} & =-\beta(t) S I
\end{aligned}
$$

The infected sub system is given by;

$$
\begin{aligned}
\frac{d E}{d t} & =\beta(t) S I-\sigma E \\
\frac{d I}{d t} & =\sigma E-\gamma I
\end{aligned}
$$

or

$$
\frac{d X}{d t}=\left(\begin{array}{c}
\beta S I \\
0
\end{array}\right)+\left(\begin{array}{l}
\sigma E \\
-\sigma E+\gamma I
\end{array}\right)
$$

where $X=(E, I)^{T}$

Linealising the system 2 about the DFE we get the Jacobian matrix

$$
\begin{aligned}
J & =\left(\begin{array}{cc}
-\sigma & \beta \\
\sigma & -\gamma
\end{array}\right) \\
& =\left(\begin{array}{cc}
0 & \beta \\
0 & 0
\end{array}\right)-\left(\begin{array}{cc}
\sigma & 0 \\
-\sigma & \gamma
\end{array}\right) \\
& =T-V
\end{aligned}
$$

where

$$
T=\left(\begin{array}{ll}
0 & \beta \\
0 & 0
\end{array}\right)
$$


and

$$
V=\left(\begin{array}{cc}
\sigma & 0 \\
-\sigma & \gamma
\end{array}\right)
$$

From which

$$
V^{-1}=\left(\begin{array}{cc}
\frac{1}{\sigma} & 0 \\
\frac{1}{\gamma} & \frac{1}{\gamma}
\end{array}\right)
$$

and the next generation matrix $(\mathrm{K})$ is given by

$$
K=T V^{-1}=\left(\begin{array}{cc}
\frac{\beta}{\gamma} & \frac{\beta}{\gamma} \\
0 & 0
\end{array}\right)
$$

which is a singular matrix hence the spectral radius equals the trace of the matrix. Thus

$$
R_{0}=\frac{\beta}{\gamma}
$$

\subsection{Epidemic Threshold}

Theorem 2.1. Let $(S, E, I, \Re)$ where $\Re=R+D$, be the solution of the system 1 in $\Omega=\left\{(S, E, I, \Re) \in \mathbb{R}_{+}^{4} \mid S, E, I, \Re \geq 0, S+E+I+\Re=1\right\}$ then if $S_{0}$ is greater than the relative removal rate $\left(\rho=\frac{\gamma}{\beta}\right)$, then the number of infected individuals will first increase to the maximum value

$$
\mathfrak{I}_{\text {max }}=S_{0}-S(t)+\rho \ln \left(\frac{S(t)}{S_{0}}\right) \quad \text { where } \mathfrak{I}=E+I
$$

and then decrease to zero as $t \longrightarrow \infty$ while $S(t)$ is a decreasing function with a limiting value $S_{\infty}$ which is a root of the equation

$$
\mathfrak{I}(0)+S(0)-S_{\infty}+\rho \ln \left(\frac{S_{\infty}}{S(0)}\right)=0
$$

Proof. Let $\Re=R+D$ be the total number of removed individuals either though death or recovery from the disease then the system 1 becomes

$$
\begin{aligned}
\frac{d S}{d t} & =-\beta(t) S I \\
\frac{d E}{d t} & =\beta(t) S I-\sigma E \\
\frac{d I}{d t} & =\sigma E-\gamma I \\
\frac{d \Re}{d t} & =\gamma I
\end{aligned}
$$


Dividing equation 3 by 6 yields

$$
\begin{aligned}
\frac{d S}{d R} & =\frac{-\beta S I}{\gamma I} \\
& =-\frac{S}{\rho}
\end{aligned}
$$

where $\rho=\frac{\gamma}{\beta}$ is the relative removal rate.

$$
\frac{1}{S} d S=-\frac{1}{\rho} d R
$$

Integrating both sides in the limits from 0 to $t$, we get

$$
\ln \left(\frac{S(t)}{S(0)}\right)=-\frac{1}{\rho} R(t)
$$

or

$$
S(t)=S(0) e^{\frac{-R(t)}{\rho}}
$$

Thus $S(t)>0 \quad \forall \quad t>0$ hence there will be susceptible individuals at the end of the epidemic i.e. the epidemic will not end due to lack of susceptible individuals.

Dividing equation 5 by 3 we get

$$
\frac{d I}{d S}=\frac{\sigma E-\gamma I}{-\beta S I}=\frac{\gamma}{\beta S}-\frac{\sigma E}{\beta S I}
$$

Dividing equation 4 by 3 , we get

$$
\begin{aligned}
\frac{d E}{d S} & =\frac{\beta S I-\sigma E}{-\beta S I} \\
& =\frac{\sigma E}{\beta S I}-1
\end{aligned}
$$

From which

$$
\frac{\sigma E}{\beta S I}=\frac{d E}{d S}+1
$$

substituting this value into equation 7 yields

$$
\frac{d I}{d S}=\frac{\gamma}{\beta S}-1-\frac{d E}{d S}
$$

or

$$
\frac{d I}{d S}+\frac{d E}{d S}=\frac{\gamma}{\beta S}-1
$$




$$
\frac{d \mathfrak{I}}{d S}=\frac{\rho}{S}-1
$$

where $\rho=\frac{\gamma}{\beta}$ is the relative removal rate and $\mathfrak{I}=I+E$ is the total number of infected individuals.

From equation 8 we note that $\mathfrak{I}$ is an increasing function of $S$ when $S<\rho$ and a decreasing function of $S$ when $S>\rho$. Since $S$ is a monotonically decreasing function then $\mathfrak{I}$ will decrease monotonically when $S<\rho$ and increase when $S>\rho$. Thus if infected individuals are introduced into a susceptible population with $S_{0}>\rho$ then the number of infected individuals will increase monotonically as $S$ decreases to the threshold value $S=\rho$ after which the number of infected individuals will decrease to zero as $t \rightarrow \infty$ (see figure 2).

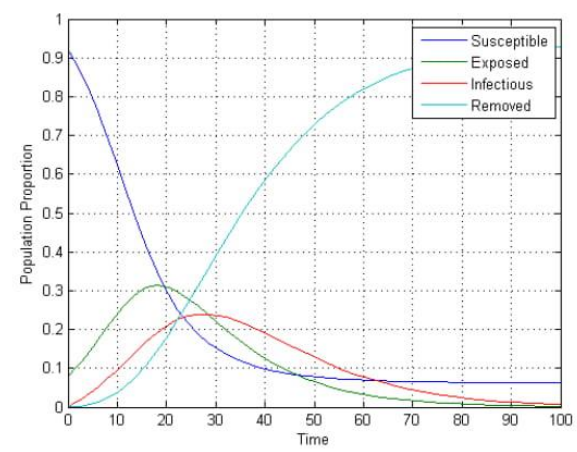

Figure 2: Trajectories of the Solution for $S_{0}=0.92, \quad E_{0}=0.08, \quad I_{0}=R(0)=0$ and $\rho=0.36$

Integrating both sides of equation 8 with respect to $S$ from zero to $t$, we get

$$
\begin{aligned}
\int_{0}^{t} d \mathfrak{I} & =\int_{0}^{t}\left(\frac{\rho}{S}-1\right) d S \\
\mathfrak{I}(t)-\mathfrak{I}(0) & =\rho \ln \left(\frac{S(t)}{S(0)}\right)-S(t)+S(0) \\
\mathfrak{I}(t) & =\mathfrak{I}(0)+S(0)-S(t)+\rho \ln \left(\frac{S(t)}{S(0)}\right)
\end{aligned}
$$

as $t \longrightarrow \infty$ the equation reduces to

$$
\mathfrak{I}(0)+S(0)-S_{\infty}+\rho \ln \left(\frac{S_{\infty}}{S(0)}\right)=0
$$

whose root $S_{\infty}$ is the number of susceptible individuals at the end of the epidemic. 


\section{Conclusion}

We observe from the above analysis that the epidemic does not end due to lack of susceptible individuals but rather it ends when there are no more infectious individuals in the population. If the ratio of the susceptible individuals $(S)$ is greater than the relative removal rate $(\rho)$ then the number of infected individuals will increase monotonically as $\mathrm{S}$ decreases to the threshold value $\rho$ after which the infected population will decay to zero as time progresses. This is demonstrated by figure 2 . In this figure, $S_{0}=0.92$ which is greater than $\rho=0.36$. It is observed that the infected population grows monotonically (red and green curves) until the susceptible population decreases to the threshold value of 0.36 after which the infected population decays to zero as a function of time. In figure 3 below $S_{0}=0.82$ which is less than $\rho=1.5$ and it is observed that the infected population decays monotonically as a function of time for all time. Figure 4 demonstrates a case where $S_{0}=\rho$ that is the initial susceptible ratio equals the relative removal rate. The results indicates that in this case the infected population cannot increase but will decay to zero as time progresses.

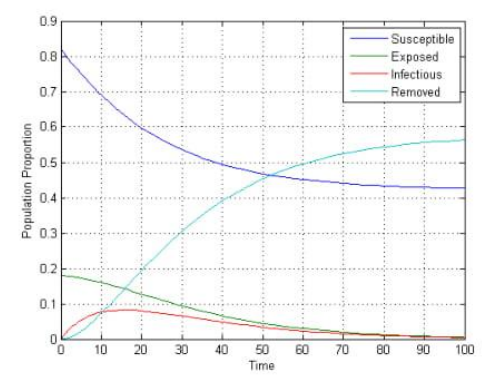

Figure 3: Trajectories of the Solution for $S_{0}=0.82, \quad E_{0}=0.18, \quad I_{0}=R(0)=0$ and $\rho=1.5$

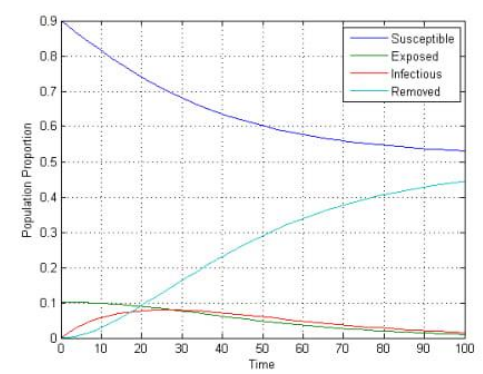

Figure 4: Trajectories of the Solution for $S_{0}=0.9, \quad E_{0}=0.1, \quad I_{0}=R(0)=0$ and $\rho=0.9$ 


\section{References}

[1] G. Chowell, N. W. Hengartner, C. Castillo-Chavez, P. W. Fenimore, and J. M. Hyman. The basic reproduction number of ebola and the effects of public health measures: the cases of congo and uganda. Journal of Theoretical Biology, 229(1):119-126, 2004.

[2] O. Diekmann and J. A. P. Heesterbeek. Mathematical epidemiology of infectious diseases: Model building, analysis and interpretation. Chichester, UK: Wiley, 2000 .

[3] O. Diekmann, J. A. P. Heesterbeek, and M. G. Roberts. The construction of the next generation matrices for compartmental epidemic models. Journal of Royal Society Interface, 7:873-885, 2010.

[4] ECDC. Outbreak of ebola virus disease in west Africa. Seventh Update, Stockholm, 2014.

[5] S. K. Gire, A. Goba, K. G. Andersen, R. S. G. Sealfon, D. J. Park, L. Kanneh, and S. Jalloh. Genomic surveillance elucidates ebola virus origin and transmission during the 2014 outbreak. Science, 345(6202):1369-1372, 2014.

[6] WHO. Ebola virus disease-fact sheet no 103. Geneva: World Health Organisation, http://www.who.int/mediacentre/factsheets/fs103/en/, 2014. 